\title{
CRITICAL HEAT FLUX IN NANOFLUIDS AT QUASI-STATIONARY AND STEPWISE HEAT GENERATION
}

\author{
Mikhail Moiseev ${ }^{1, *}$, Vladimir Zhukov ${ }^{1}$, and Denis Kuznetsov ${ }^{1}$ \\ ${ }^{1}$ Kutateladze Institute of Thermophysics SB RAS, Novosibirsk, Russia
}

\begin{abstract}
In this paper results of an experimental study on critical heat flux in nanofluid at quasi-stationary and stepwise heat generation are presented. Freon R21 with addition of 0.0077 vol. $\%$ of $\mathrm{SiO}_{2}$ nanoparticles was used as test fluid. Boiling curves, critical heat fluxes and temperatures of boiling initiation were obtained for pure fluid and for nanofluid. It was shown that the addition of nanoparticles didn't affect heat transfer at pool boiling, but critical heat fluxes at quasi-stationary and stepwise heat generation were increased.
\end{abstract}

\section{Introduction}

Boiling is an effective way to remove heat from the surface that is widely used in various technical applications. The increasing requirements for efficiency and miniaturization of equipment give an impetus to the search of methods to intensify heat transfer during boiling and to increase critical heat flux (CHF) $[1,2]$. In recent decades, one of the promising trends in enhancing the boiling characteristics is the use of nanofluids. The addition of nanoparticles can significantly increase both the heat transfer coefficient and CHF [3]. However, the obtained results are often contradictory and depend on many factors, so the practical use of nanofluids needs further systematic research. In addition, there is a necessity in the experiments to be carried out in nanofluids under non-stationary heat release and to simulate different emergency processes (depressurization of devices, removal of rods in nuclear power plants, etc.). At present there are only few studies on the topic. The available works demonstrate an increase in non-stationary CHF when adding nanoparticles $[4,5]$.

This work experimentally investigates boiling of Freon R21 with addition of 0.0077 vol. $\%$ of $\mathrm{SiO}_{2}$ nanoparticles at quasi- and non-stationary heat release.

\section{Description of the experiment}

The experiments were carried out in a large volume of Freon R21 with addition of 0.0077 vol. $\%$ of $\mathrm{SiO}_{2}$ particles with a size of $20-25 \mathrm{~nm}$. The horizontal cylindrical pipe of stainless steel with a length of $50 \mathrm{~mm}$, an outer diameter of $3 \mathrm{~mm}$ and a wall thickness of $0.5 \mathrm{~mm}$

\footnotetext{
*Corresponding author: moiseevmikhail@gmail.com
} 
was used as a working section. Heating of the section was performed by a controllable constant current source. The temperature of the wall and liquid in bulk under quasistationary conditions was determined by thin-film platinum resistance thermometers HEL700. At non-stationary heat release the wall temperature was determined from the numerical solution of the heat equation. The experiments were conducted in the range of reduced pressures $P / P_{c r}=0.037-0.056(P=0.19-0.29 \mathrm{MPa})$. Detailed description of the setup is given in [6].

Experiments were carried out at two regimes of heat release. For the first regime, which we call "quasi-stationary heat release", the heat flux was increased by small amount step by step, and each time, stabilization of wall temperature was waited for. For the second regime, which we call "stepwise heat release", the heater was stepwise supplied with a constant current of $420 \mathrm{~A}$. In this case the fluid that initially was at saturation temperature can reach high level of metastability, and development of boiling crisis changes drastically.

\section{Results of the experiment}

Fig. 1 shows the boiling curves in pure fluid and in nanofluid during the quasi-stationary heat release. The addition of the nanoparticles almost did not influence the efficiency of heat transfer. However, overheating of the wall, in which there is a transition from the region of single-phase convection to boiling incipience, decreased from $51 \mathrm{~K}$ to $25 \mathrm{~K}$.

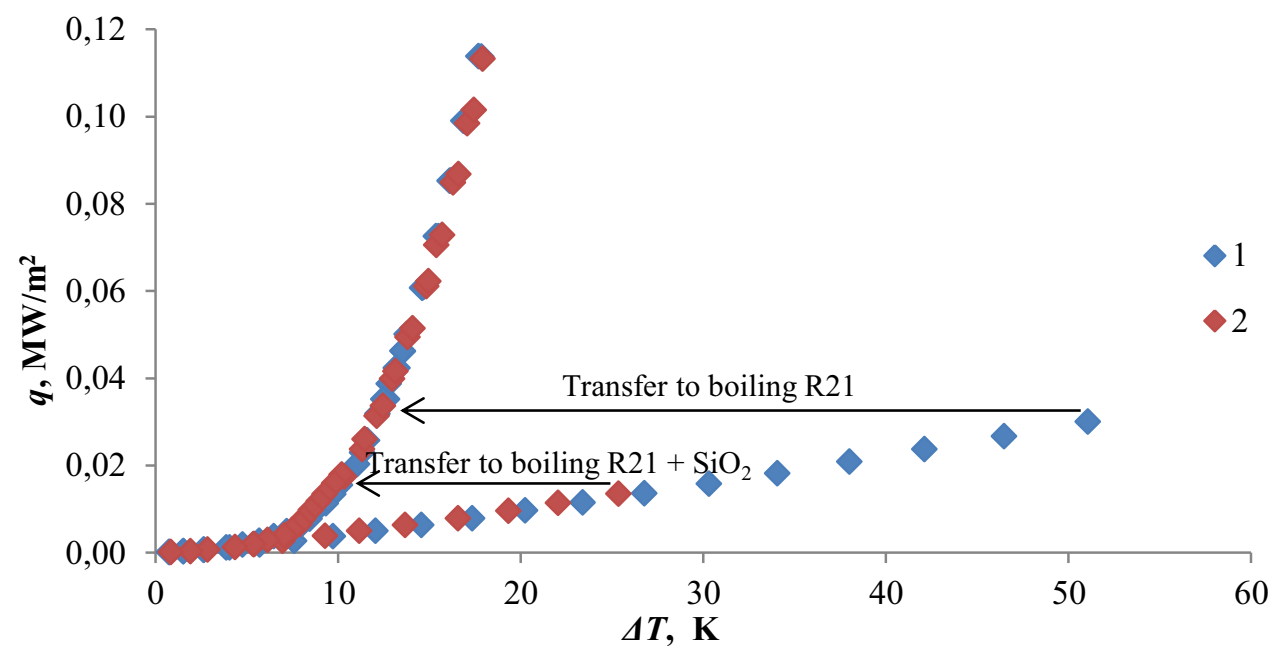

Fig. 1. Boiling curves. $1-\mathrm{R} 21 ; 2-\mathrm{R} 21+0.0077 \% \mathrm{SiO}_{2} . P / P_{c r}=0.037$.

Experiments were carried out to determine CHF. In the pre-crisis region the electric current through the heater was gradually increased by a small value, and each time, stabilization of the wall temperature was waited for. The achievement of the CHF was determined visually by vapor film forming. The results of the experiments are presented in fig. 2 . Experimental data for pure fluid and nanofluid are well described by Kutateladze formula:

$$
q_{\mathrm{cr}}=k r \sqrt{\rho_{\mathrm{v}}}\left[g \sigma\left(\rho_{\mathrm{l}}-\rho_{\mathrm{v}}\right)\right]^{1 / 4},
$$

where $k$ is empirical coefficient, $r$ - latent heat of evaporation, $g$ - gravitational acceleration, $\sigma-$ surface tension, $\rho_{v}-$ vapor density, and $\rho_{1}-$ liquid density.

Empirical coefficients were found from experimental data using least squares method. For the nanofluid $k=0.152$, and for pure Freon R $21 k=0.137$. Thus, the presence of 
0.0077 vol. $\%$ of $\mathrm{SiO}_{2}$ nanoparticles has led to the increase in the value of the first CHF by $11 \%$.

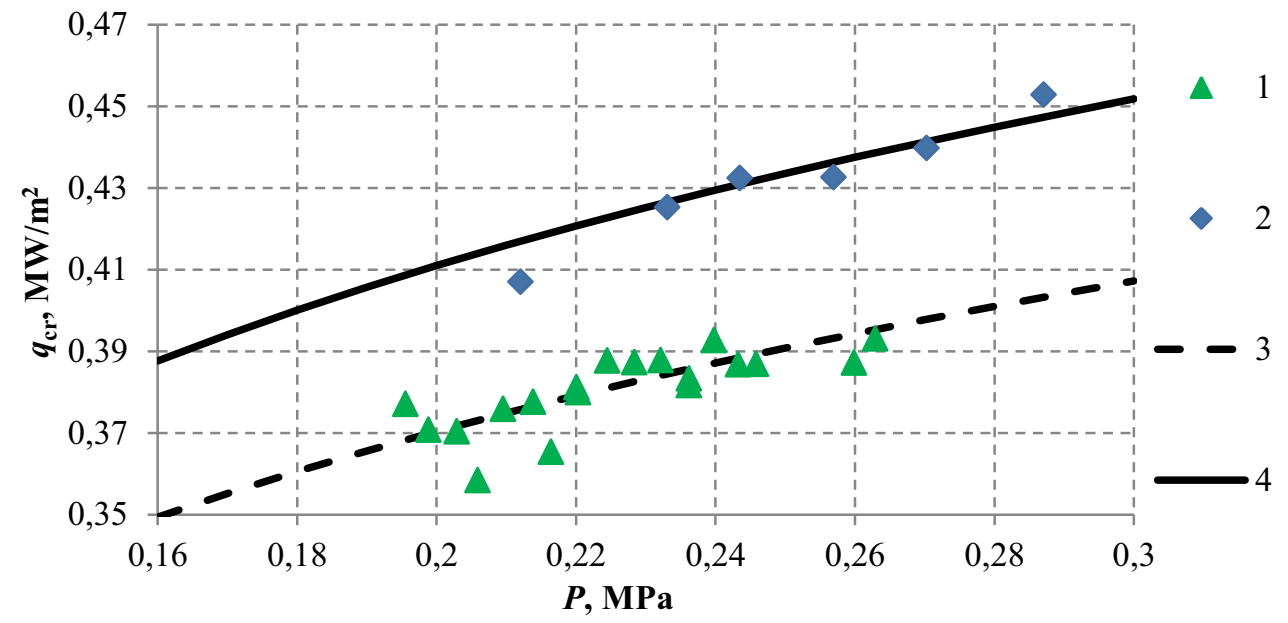

Fig. 2. The dependence of the critical density of the heat flux on pressure. Experimental data: $1-$ Freon R21; 2 - Freon R21 $+\mathrm{SiO}_{2} ;$ Calculation by Kutateladze $\boldsymbol{q}_{\boldsymbol{c r}}=\boldsymbol{k} \boldsymbol{r} \sqrt{\boldsymbol{\rho}_{\boldsymbol{v}}}\left[\boldsymbol{g} \boldsymbol{\sigma}\left(\boldsymbol{\rho}_{\boldsymbol{l}}-\boldsymbol{\rho}_{\boldsymbol{v}}\right)\right]^{\mathbf{1 / 4}}: 3$ $-k=0.137 ; 4-k=0.152$.

In the experiments under non-stationary heat release, the heater was stepwise supplied with a constant current of $420 \mathrm{~A}$. The rate of the wall heating in this case was $2180 \mathrm{~K} / \mathrm{s}$, and overheating relative to the saturation temperature reached $70 \mathrm{~K}$. An increase in the wall temperature results in an increase in the density of the heat flux, transferred to the liquid by thermal conductivity, since for the duration of an experiment (about $30 \mathrm{~ms}$ ) the convection has no time to develop. The interface of a steam bubble growing at the nucleation site becomes hydrodynamically unstable when a certain wall overheat is reached. This leads to the initiation of evaporation front propagation along the heater (in the layer of metastable liquid) and the onset of the heat transfer crisis.

Fig. 3 shows the dependence of the heat flux density $q$, transmitted by the wall to the fluid, on the temperature of the wall overheating $\Delta T$. The value of $q$ was obtained from the numerical solution of the heat equation. Vertical lines mark the minimum temperatures of the wall, at which the evaporation front propagation was recorded. In pure Freon R21, the corresponding heat flux density amounted to $0.146 \mathrm{MW} / \mathrm{m}^{2}$ ( 3 times less than CHF at quasi-stationary heat release). In nanofluid $\mathrm{R} 21+\mathrm{SiO}_{2}$, it is equal to $0.184 \mathrm{MW} / \mathrm{m}^{2}$. Thus, the addition of $\mathrm{SiO}_{2}$ nanoparticles has led to a $26 \%$ increase in CHF at stepwise heat release. 


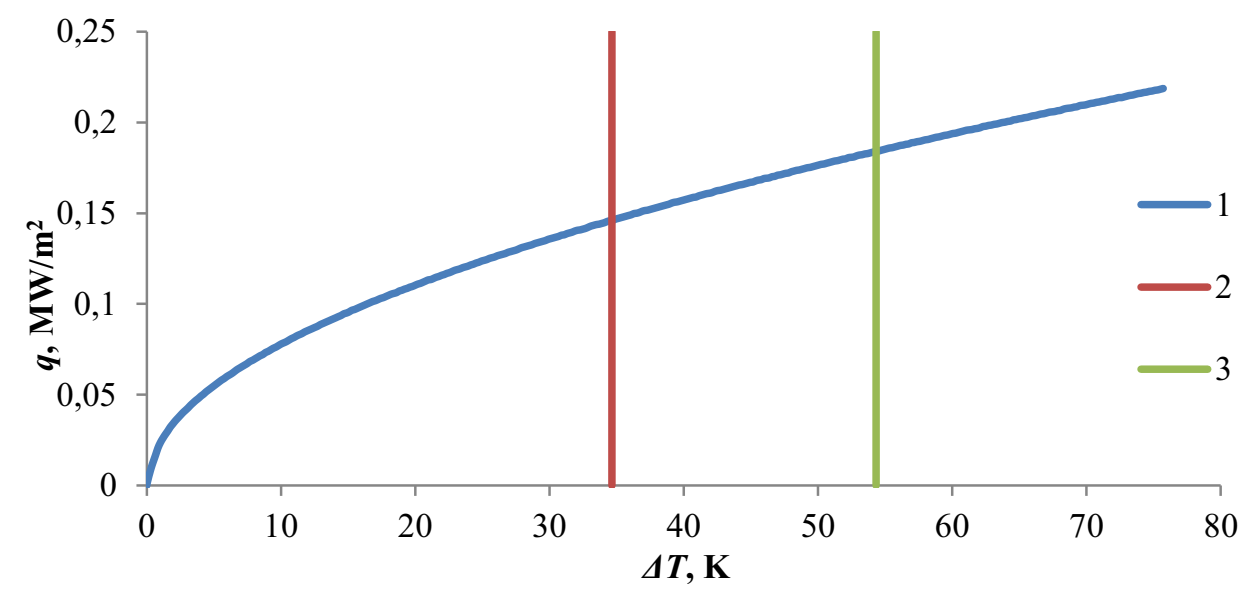

Fig. 3. 1 - heat flux at solid-liquid interface; 2 - front initiation in Freon R21; 3 - front initiation in nanofluid $\mathrm{R} 21+0.0077$ vol. $\% \mathrm{SiO}_{2}$. $\mathrm{P} / \mathrm{P}_{\mathrm{cr}}=0.037$.

\section{Conclusions}

Adding 0.0077 vol. $\%$ of $\mathrm{SiO}_{2}$ nanoparticles in Freon $\mathrm{R} 21$ has not noticeably influenced the heat transfer coefficient during boiling, but has led to a decrease in boiling-up temperature. $\mathrm{CHF}$ in nanofluid under quasi-stationary heat release increased by $11 \%$ compared to pure fluid.

CHF at stepwise heat release was 3 times lower than CHF in quasi-stationary regime. Addition of nanoparticles provided 26\% increase in CHF under non-stationary heat release. Wall overheat corresponding to boiling initiation also increased from 35 to $55 \mathrm{~K}$.

\section{Acknowledgment}

The work was financially supported by RFBR (project No. 13-08-00178).

\section{References}

1. S.Y. Misyura, Exp. Therm. Fluid Sci. 75, 43 (2016)

2. V.E. Nakoryakov, and S.Y. Misyura, JET 25(1), 24 (2016)

3. D. Ciloglua, and A. Bolukbasib, App. Therm. Eng. 84(5), 45 (2015)

4. V.I. Sharma, J. Buongiorno, T. McKrell, and L. Hu, Int. J. Heat Mass Transfer 63, $425(2013)$

5. L. Fan, J. Li, D. Li, L. Zhang, Z. Yu, and K. Cen, Int. J. Therm. Sci. 91, 83 (2015)

6. A.N. Pavlenko, A.A. Tairov, V.E. Zhukov, A.A. Levin, and M.I. Moiseev, JET 23(3), 173 (2014) 